\title{
Visible, potential, and functional land degradation assessment in southern Africa
}

\author{
Zidong $\mathrm{Li}^{1}$, Shuai Wang ${ }^{1}$, Dexin $\mathrm{Gao}^{1}$, Yanxu Liu ${ }^{1}$, Walter Musakwa ${ }^{2}$, Fangli Wei ${ }^{3}$, \\ YueJun Wang ${ }^{4}$, and Darifu $\mathrm{Ba}^{4}$ \\ ${ }^{1}$ Beijing Normal University Faculty of Geographical Science \\ ${ }^{2}$ University of Johannesburg \\ ${ }^{3}$ Research Center for Eco-Environmental Sciences, Chinese Academy of Sciences \\ ${ }^{4}$ Hetao College
}

March 10, 2021

\begin{abstract}
Land degradation in drylands threatens vulnerable ecosystems and socioeconomic development. Currently, NDVI derived from remotely sensed data is the main tool used for degradation detection. Three indicators have usually been used to detect land degradation: (1) NDVI trends for visible degradation, (2) residual for human-induced potential degradation, and (3) sensitivity of vegetation to rainfall for functional degradation. However, few studies have integrated and compared these three indicators. In this study, we used Global Inventory Monitoring and Modelling System Version (GIMMS 3.1) NDVI dataset and Multiple Source Weighted-Ensemble Precipitation (MSWEP) rainfall dataset (1982-2015) and applied linear regression, Time Series Segmented and Residual Trend (TSS-RESTREND), and Sequential Regression (SeRGs) methods to detect degradation in southern Africa. The results showed that degradation was detected by these three respective indicators in $18.7 \%, 11.3 \%$, and $7.1 \%$ of the study area. Degradation from any one type was found to occupy $27.21 \%$ of the total area, whereas the co-occurrence of two or more types only occupied $3.84 \%$. These results indicate the dominant discrepancies among these indicators and the independent relationships among the degradation processes. Despite significantly greening, potential degradation and functional degradation were still observed. On the regional scale, spatial patterns of degradation were affected by different levels of aridity. On the national scale, the proportions of degradation were still influenced by increased population, inadequate policies, and other factors. This study highlights the need to detect degradation with multiple indicators and improves our understanding of degradation types and intensity.
\end{abstract}

\section{Hosted file}

Manuscript.pdf available at https://authorea.com/users/400586/articles/512892-visiblepotential-and-functional-land-degradation-assessment-in-southern-africa 